\title{
The differences in waveform between photoplethysmography pulse wave and radial pulse wave in movement station
}

\author{
Kun Li, Song Zhang ${ }^{*}$, Lin Yang, Zhichang Luo and Guanxiong Gu \\ College of Life Science and Bio-Engineering, Beijing University of Technology, Beijing 100124, \\ China
}

\begin{abstract}
Objective: There are many similarities between the photoplethysmography(PPG) pulse wave and the radial pulse when a body is in a stationary state, but the difference between them under conditions of movement is not yet clear. Finding these differences may help further understanding of the cardiovascular system. Methods: PPG and radial pulse wave were recorded simultaneously while subjects were conducting a bicycle exercise test that included the resting and exercise state, while the $\mathrm{K}$ and $\mathrm{K}$ ' parameters were being acquired from the PPG and radial pulse, respectively. Furthermore, the pulse objective pattern is observed via the time domain waveform and XY graph. Results: When the body's state of movement changes dramatically, there is a time difference between the pulse parameter $\mathrm{K}$ and the pulse amplitude, and the difference of the pulse pattern is enhanced during the exercising phase. Conclusion: Radial pulse waves are not the same as PPG during exercise in either the pulse parameter or the pulse pattern. This information can be used to further evaluate the state of arterial circulation and microcirculation.
\end{abstract}

Keywords: photoplethysmography(PPG), radial pulse wave, waveform parameter, waveform pattern

\section{Introduction}

Until now, pulse waves could be used to calculate cardiovascular parameters noninvasively, such as stroke volume (SV), cardiac output (CO), total peripheral resistance (TPR), blood flow alternate (ALK), and so on [1]. As the two main types of pulse wave, radial pulse wave and photoplethysmography(PPG) pulse wave are both widely used in to evaluate cardiovascular function. Furthermore, PPG reflects the status of the human body microcirculation while the radial pulse wave reflects the status of arterial circulation. Since microcirculation is a part of arterial circulation, there are considerable similarities between the two. There are studies found that PPG and radial artery pulse wave are highly consistent in reflecting the cardiovascular blood flow status in quiet conditions [1]. However, the differences that exist between the two kinds of pulse wave while in the movement station remain unclear.

Related research, including the research of carotid - wrist artery cross-correlation modal [2] and the

\footnotetext{
*Corresponding author: Song Zhang, College of Life Science and Bio-Engineering, Beijing University of Technology, Beijing 100124, China. Tel.: +8613901218968; Fax:+8667392010; E-mail: Zhangsong@bjut.edu.cn.
} 
research of pulse wave velocity (PWV) in different parts of the body [3], have shown that pulse waves can propagate along the aorta without any significant distortion in certain cases. Other studies have suggested that the cardiovascular dual elastic chambers model theory can be expanded in the field of microcirculation by modeling the microcirculation volume pulse blood flow through the fingers, and microcirculation model parameters can be calculated from the measured finger volume pulse waveform, which strongly suggests that the radial artery pulse wave has an exact relationship with the finger volume pulse wave [4,5], which can probably replace the former due to the simplicity of the PPG test. However, since arterial circulation is a dynamic elastic system, the relationship between the radial pulse wave and PPG may change rapidly during exercise. If so, further cardiovascular information can be acquired from the differences between them.

Here, the pulse wave parameter K [1] is adopted. It reflects the condition of peripheral vascular resistance, defined as Eq. (1).

$$
K=\frac{P_{m}-P_{d}}{P_{s}-P_{d}}
$$

Among them,

$$
P_{m}=\frac{1}{T} \int_{0}^{T} P(t) d t
$$

$P_{m}$ is the mean value of the radial pulse wave pressure $P(t)$ in a cardiac circle, and $P s$ and $P d$ represent systolic blood pressure and diastolic blood pressure, respectively, as Figure 1 illustrates.

Here, the K value of the radial pulse wave and K' of PPG is calculated during exercise, and then any differences that exist between the two types of pulse wave in their amplitude and pulse objective pattern are observed.

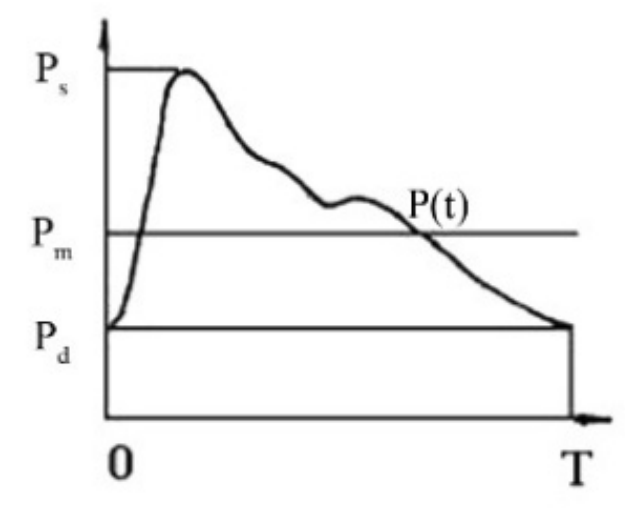

Fig. 1. Characteristic parameter $\mathrm{K}$ of pulse wave. 


\section{Materials and methods}

\subsection{Experimental subjects}

The experiments were carried out in accordance with the Declaration of Helsinki (2000) of the World Medical Association by requiring informed consent from the experimental subjects and protocols approved by the Institutional Research Review Board at Hospital of Beijing University of Technology. 55 volunteers were observed: 51 male and 4 female. The average age of the volunteers was $19.8 \pm 0.8$. Some of the volunteers suspended the experiment due to extreme fatigue, difficulty breathing, dizziness, tinnitus, nausea, chest pain, paleness, or trembling. In the end, 44 cases of effective data were collected.

Radial pulse wave and PPG were acquired, respectively, by a radial artery pressure pulse wave sensor (China, Beijing Yes) and a finger volume pulse wave sensor (China, Beijing ChaoSi). The data was transferred to a PC through a PowerLab5 data recording and analysis system (Australia, ADInstruments). Exercise load was provided by an Ergomedic 839E cycle ergometer (Sweden, Monark).

Pulse wave was analyzed using LabChart 7 Pro (Australia, ADInstruments) and pulse wave analysis software (China, Beijing University of Technology).

\subsection{Experimental procedures}

In the study, the power meter treadmill test method was taken as our experimental method, which was modified according to the experimental request based on the Bruce test [6]. The experiment is separated into 3 parts, including the stationary phase, exercise phase, and recovery phase. Throughout the whole experimental process, the radial pulse wave, PPG, heart rate, and ECG were recorded in real-time, while the blood pressure was only taken once a minute. The stationary phase lasts for 1 minute, during which the subjects remain static. During the exercise phase, the subjects began cycling on the cycle ergometer with an initial load of 50 Watts, and the load was then increased by 25 Watts every 3 minutes until the subjects' heart rates rose to 170 beat $/ \mathrm{min}$ [7]. The subjects then stopped cycling and entered the recovery phase. The recovery phase gives subjects a rest period, during which the pulse wave changes could be observed.

\subsection{Data processing}

$\mathrm{K}$ and $\mathrm{K}$ ' of the radial pulse wave and PPG were calculated every 5 seconds with an equalization of every 20 data points being made, and the trace curve of these parameters being plotted over time. On the other hand, the pulse wave's pulse objective pattern was compared between the two kinds of pulse wave. Additionally, to further compare the pulse waves, an XY graph was plotted with the radial pulse wave's homogenized amplitude as the X-axis, and the PPG's homogenized amplitude as the Y-axis. The amplitude of the two kinds of pulse wave was calculated every 5 seconds in order to observe the changing trend and to convert the pulse amplitude and rate to the unit size.

\section{Results}

\subsection{K and $K^{\prime}$}

The variation trends have many things in common in the trace curve of $\mathrm{K}$ and $\mathrm{K}$ ' over time. Take 
subjects No.31 and No.23 as an example, as are shown in Figures 2 and 3. The whole experimental process can be divided into 4 parts in accordance with the changing characteristics of K and K': Inexercise drop down period, After-exercise decline period, After-exercise rapidly rising period and After-exercise slow rising period.

From the second to fourth period is the period in which the subjects stop exercising and begin resting, the curve of $\mathrm{K}$ and $\mathrm{K}$ ' over time both initially decrease and then eventually increase. The both extreme points of $\mathrm{K}$ and $\mathrm{K}$ ' fall behind the time point of the moving-stop point, and the extreme point falls further behind $\mathrm{K}$ over time.

\subsection{Pulse objective pattern}

The characteristic points are shown in Figure 4. During the whole experimental process, point A (main wave) rises rapidly, while point B (tidal wave) disappears, with point D (dicrotic wave trough) and point $\mathrm{C}$ (dicrotic wave peak) falling, among which point $\mathrm{D}$ falls under the line $\mathrm{OE}$ (the baseline of the radial pulse wave). In the recovery phase, the changing trend of the characteristic point is contrary to the exercise phase. PPG's characteristic point changes in the same way. However, point D is always over the baseline OE. Take subject No. 2 as an example, shown below in Figure 5.
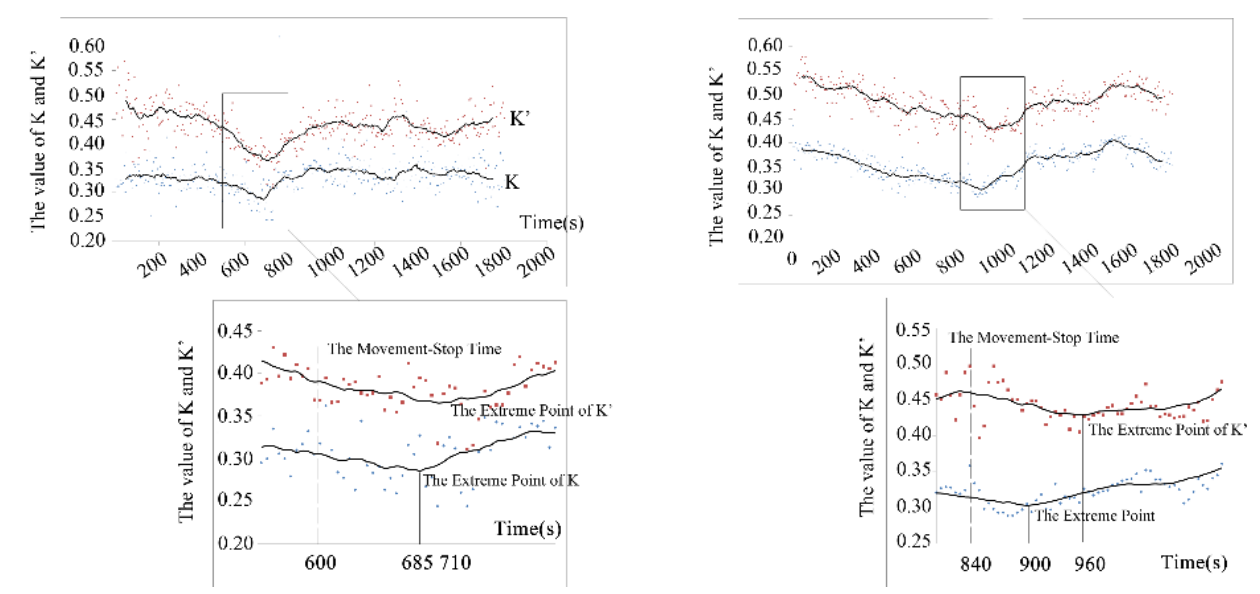

Fig. 2. The $\mathrm{K}$ and $\mathrm{K}$ ' trends of 31 subjects over time. Fig. 3. The $\mathrm{K}$ and $\mathrm{K}$ ' trends of 23 subjects over time.

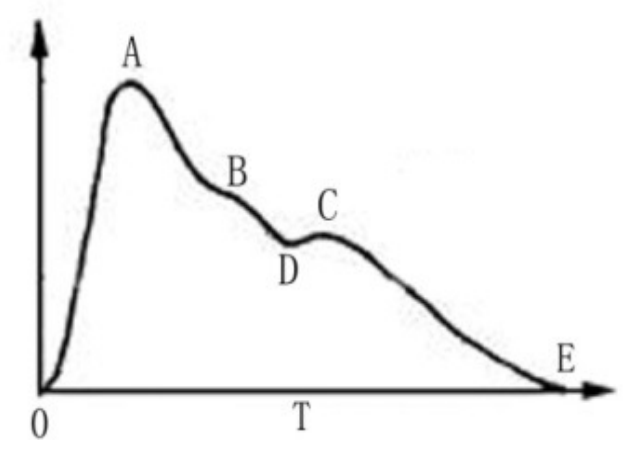

Fig. 4. Characteristic point of pulse wave.

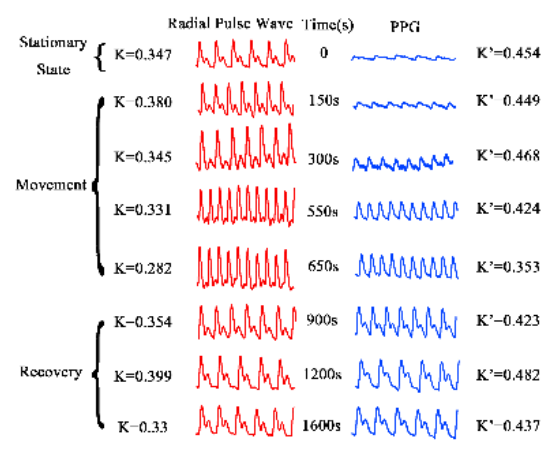

Fig. 5. The radial pulse wave and PPG of Subject No. 2 during the experiment. 

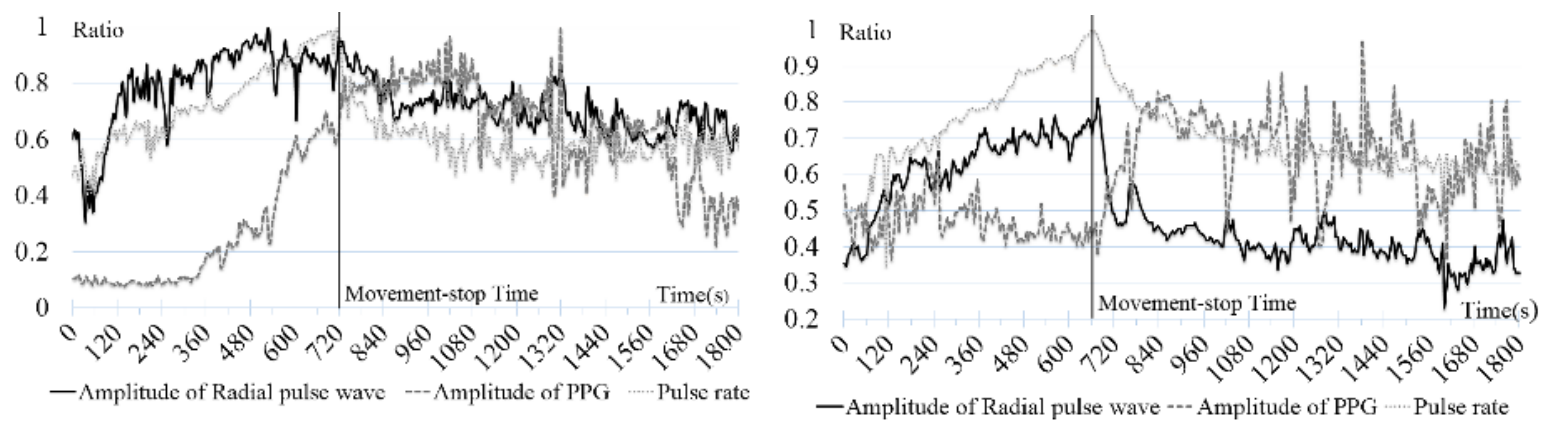

Fig. 6. Subject No.2's pulse rate and waveform amplitude. Fig. 7. Subject No.10's pulse rate and waveform amplitude.

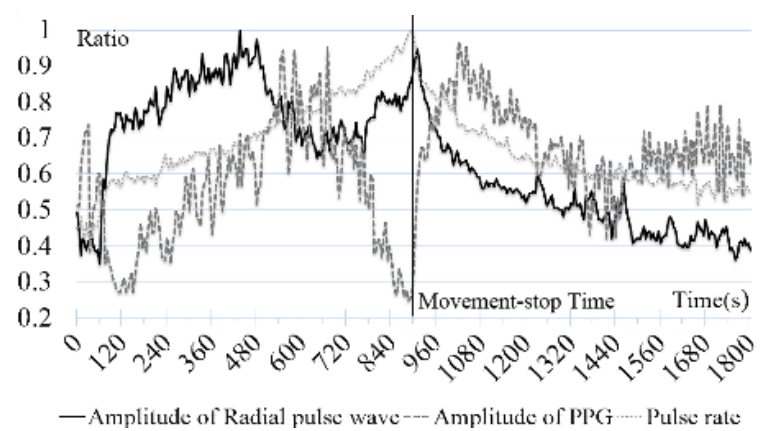

Fig. 8. Subject No.12's pulse rate and waveform amplitude.

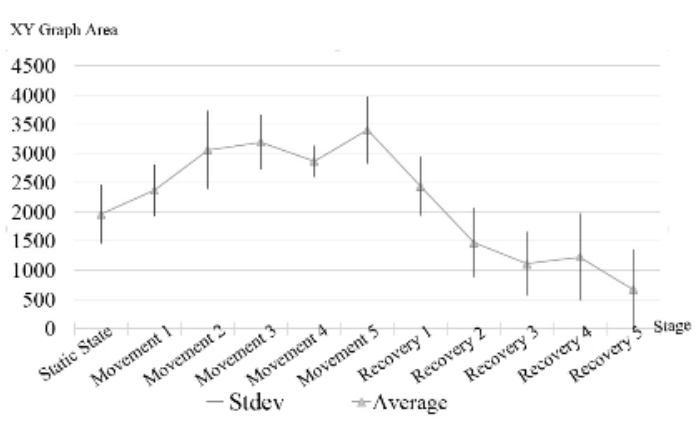

Fig. 9. The XY graph of Subject No.14 during the experiment.
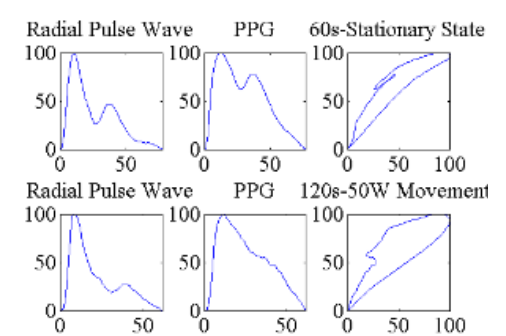

Radial Pulse Wave PPG 300s-75W Movement

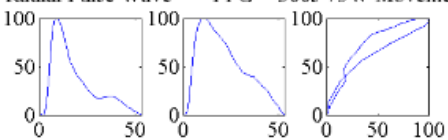

Radial Pulse Wave PPG 480s-100W Movemen

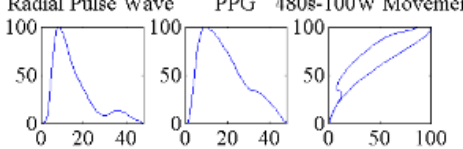

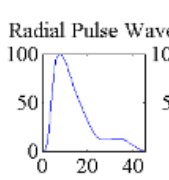

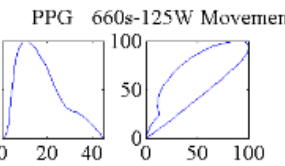

Radial Pulse Wave PPG 840s-150W Movemen
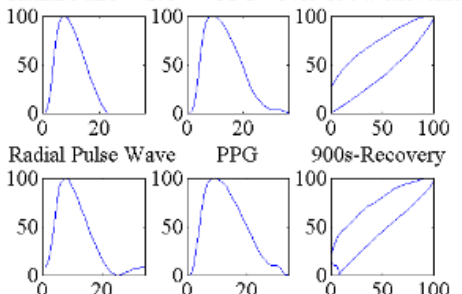

900 s-Recovery
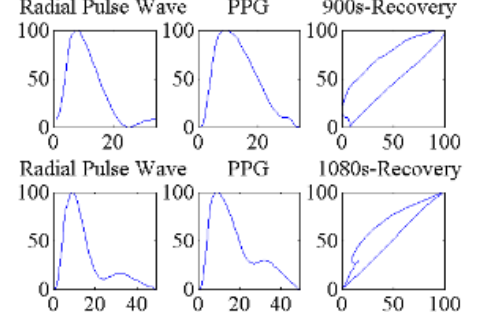

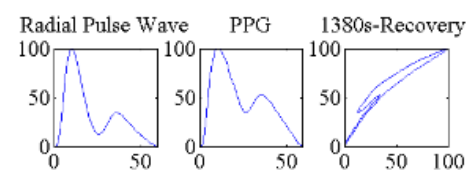

Radial Pulse Wave PPG 1680s-Recovery

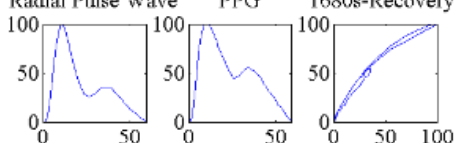

Radial Pulse Wave PPG 1800s-Recovery

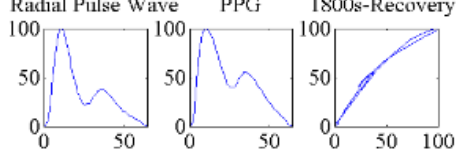

Fig. 10. The XY mean phase area of all subjects during the experiment. 
In Figures 6-8 the pulse wave's amplitude during the experiment appears to be distinct, among which PPG's amplitude increases after the movement-stop point (where pulse rate drop instantly), while the radial pulse wave's amplitude increases during exercise and decreases consistently during recovery. That is to say, the amplitude of PPG changes after the radial pulse wave does.

In the XY graph, the curve forms a circle, which is counterclockwise from the beginning of the pulse wave to the end by time. For example, observe subject No. 14 in Figure 9. The circle changes in shape and size by exercise and recovery. To be specific, the circle increases in size during the exercise phase and then drops during the recovery phase, which is reflected in the circle's area. The time span of exercise and recover process is normalized first since not all subjects conduct a same-time experiment, with the two phase being divided respective into 5 parts and the mean area of all subjects at the corresponding normalized-moment was calculated, shown in Figure 10.

\section{Discussion}

In this study, it was demonstrated that the radial pulse wave and PPG are quite different in the movement state in terms of the pulse wave parameter, pulse objective pattern, and XY circle area, which confirms the assumption made before in the article. $\mathrm{K}$ and $\mathrm{K}$ ' change similarly with time, while $\mathrm{K}$ ' falls behind $\mathrm{K}$ throughout the entire experiment. As for the pulse objective pattern, it was observed that the pulse amplitude is the main difference between the two kinds of pulse wave; furthermore, PPG falls behind the radial pulse wave in amplitude with time, as well. Moreover, the dicrotic wave trough of the radial pulse wave changes more evidently than PPG's does when the subjects endure strenuous exercise.

The $\mathrm{K}$ and $\mathrm{K}$ ' appears distinct at the time at which the extreme point is reached, which represents a different physical state. Subject No. 31 stops exercising at 840s, and 60s later (named delta-T1), the extreme point of K appears. Furthermore, another 60s later (named delta-T2), the extreme point of K' appears. Similarly, subject No. 23's two delta-Ts are, respectively, $85 \mathrm{~s}$ and $25 \mathrm{~s}$, of which delta-T1 is longer than subject No.31's whereas delta-T2 is shorter. The mean delta-T1 of all subjects is $19 \pm 40 \mathrm{~s}$, and mean delta-T2 is $37 \pm 53 \mathrm{~s}$, so we can see the time-delay exist extensively. As for the differences between subject No. 31 and subject No. 23, it is thought that delta-T1 represents the response speed of motion state change, while delta-T2 represents the speed of the microcirculation's relative response speed of motion state change, thus subject No. 31 is better in the aspect of arterial circulation than subject No. 23, but inferior in microcirculation. Therefore, delta-T1 and delta-T2 may be able to help us evaluate the state of both arterial circulation and microcirculation.

The dicrotic wave trough of the two kinds of pulse wave decreases in exercise conditions for the following listed reasons. The systole is more acute, and the blood flow rate is higher in the movement state. When the heart is in diastole, the blood goes forward persistently, thus causing the vessel to be kept in low tension, which makes the radial pulse wave (representing the pressure) and PPG (representing the volume) decrease further. Additionally, the pressure can be negative, but the volume must be positive. Some subjects did not exhibit identical character in the dicrotic wave trough, with the dicrotic wave trough of the radial pulse wave not coming below the baseline, which indicates worse vascular elasticity. According to the conclusion that the dicrotic wave is the superposition of the pulse wave and reflected wave, the blood vessels should deform in the opposite direction of the blood pressure if the vascular elasticity is enough to strengthen the reflected wave.

When human body is in movement, there are three ways to increase the cardiac output, respectively are heart rate, blood pressure and vasodilatation. Heart rate rises in order to increase the bump-blood 
times in unit time span, blood pressure increases the blood volume of a single heart pump out, and vasodilatation reduced the blood-flow resistance. Hearts' compensatory responses in heart rate and blood pressure are common phenomenon in the crowd, however, the best way is vasodilatation which allows heart bump more blood at the same time the heart will get more rest. Furthermore, the cardiovascular potential of an athlete depends mostly on the ability of vasodilatation which is shown most distinct as the blood flow in microcirculation. Amplitude reflects the pulse pressure and the maximum blood flow in microcirculation, respectively, for the radial pulse wave and PPG. During the exercise phase, heart rate increases steadily until exercise stops. The amplitude of the radial pulse wave increases simultaneously, causing the vessel pressure to increase. The PPG amplitude, however, changes in three different ways, which reflects the different microcirculation status in the movement process. The three ways represent the transition from the best diastolic ability to the worst: for the first, the PPG amplitude increases representing the vasodilatation with the exercise duration and lasts until the early recovery; the second increases initially and then drops at the end of the exercise phase expressing the ability of vasodilatation decrease or not good enough ensure the peripheral circulation; and the other decreases until the movement-stop point and then increases after movement stopping which is because during exercise the internal organs and muscles need more blood, if the diastolic ability is poor there is not enough blood in peripheral vascular through your fingers.

The XY graph shows the overall difference [8] between the pulse objective pattern of the radial pulse wave and PPG. The greater the difference is, the larger the circle's area. Thus, the difference is enhanced during exercise and is later reduced in the recovery phase. A phenomenon of the circle-area is that the area becomes negative in the recovery phase. This may be due to the fact that the PPG increases are more drastic in the OA stage (as shown in Figure 4) than in the radial pulse wave, thus reflecting that the microcirculation's filling is greater than the vessel's mechanical deformation in the recovery phase.

Athletes' cardiovascular conditions and sports potential can be further evaluated according to the differences described above. Furthermore, delta-T1 and delta-T2 can be used to evaluate the response rate of arterial circulation and microcirculation, and the amplitude of PPG in the exercise phase can be used to assess vascular elasticity. However, the applications of the XY area and the dicrotic wave need to be further explored.

However, there are deficiencies in the experiment, which is only four female subjects in results, which may only show the changes in male.

\section{Conclusion}

In summary, parameters $\mathrm{K}$ and $\mathrm{K}$ ' were calculated to find the difference between the response rate of the radial pulse wave and PPG, which reflects the state of arterial circulation and microcirculation under the condition of movement. It was further found via pulse objective pattern that the amplitude of PPG follows the radial pulse wave in changing trend and that exercise can enhance the differences between the radial pulse wave and PPG on patterns. Furthermore, it was discovered that the dicrotic wave trough of the radial pulse wave is unique from PPG's.

The state of arterial circulation and microcirculation can be further evaluated according to the differences listed above. However, more characteristics are needed to describe the physical state of the cardiovascular system. 


\section{References}

[1] L. Yang, S. Zhang and Y. Yang, Study of pulse wave velocity noninvasive detecting instrument based on radial artery and finger photoplethysmography pulse wave, International Symposium on Intelligent Information Technology Application Workshops, 2008, 705-708.

[2] M. Huotari, A. Vehkaoja, K. Maatta and J. Kostamovaara, Arterial pulse wave analysis based on PPG and EMFi measurements, Electronics Conference, 2012, 187-190.

[3] H.-T. W., C.-H. L., T.-C. W. and A.-B. L., Endothelium function assessment with radial pulse wave signals, Engineering in Medicine and Biology Society, Annual International Conference of the IEEE Engineering in Medicine and Biology Society, 2009, 3035-3038.

[4] J. Allen and A. Murray, Comparison of regional variability in multi-site photoplethysmographic pulse wave characteristics, First International Conference on Advances in Medical Signal and Information Processing 476 (2000), 26-31.

[5] J.Y. Foo, S.J. Wilson and P. Wang, Factors that affect pulse wave time transmission in the monitoring of cardiovascular system, J. Clin. Monit. Comput. 22 (2008), 141-147.

[6] S.P. Linder, S. Wendelken and J. Clayman, Detecting exercise induced stress using the photoplethysmogram engineering in medicine and biology society, IEEE Engineering in Medicine and Biology Society, 2006, 5109-5112.

[7] H. Lee, W. Wang, S.-W. L., B. Wu and L.-W. K., Home-based mobile cardio-pulmonary rehabilitation consultant system, engineering in medicine and biology society, Annual International Conference of the IEEE Engineering in Medicine and Biology Society (EMBC), 2011, 989-992.

[8] A. Nazari, D. Krithivasan, S.S. Pradhan, A. Anastasopoulos and R. Venkataramanan, Typicality Graphs and their Properties, Information Theory Proceedings, 2010, 520-524. 